\title{
On Functions with Almost Periodic or Almost Automorphic First Differences
}

\author{
I. D. BERG \\ Communicated by Maurice Heins
}

Introduction. In this note we consider conditions on first differences of a function which imply uniform continuity, almost automorphy or almost periodicity of the function.

We consider throughout this note continuous bounded complex-valued functions of one real variable and denote the algebra of such functions by $C(\mathbf{R})$.

We denote by $R(\mathrm{R})$ the subspace of $C(\mathrm{R})$ consisting of real-valued functions.

If $f \varepsilon C(\mathbf{R})$ and $t$ is a real number we define $\Delta_{t} f$ by $\Delta_{t} f(x)=f(x+t)-f(x)$.

Our principal result is the following:

Let $f \varepsilon C(\mathbf{R})$. Let $T$ be a dense subset of $\mathbf{R}$ such that if $t \boldsymbol{\varepsilon} T$ then $\Delta_{t} f$ is uniformly continuous. Suppose that there is a non-zero $t_{0} \varepsilon T$ such that $\Delta_{t_{0}} f$ is almost automorphic. Then $f$ is uniformly continuous.

A theorem of Bochner's then shows us that such a function $f$ is itself almost automorphic. If in addition $\Delta_{t} f$ is almost periodic then $f$ is almost periodic.

We are then able to prove the following corollary.

Let $f \varepsilon C(\mathbf{R})$. Let $T$ be a dense subset of $\mathbf{R}$ such that if $t \varepsilon T$ then $\Delta_{t} f$ is uniformly continuous. Suppose that there is a real non-zero $s$ such that $\Delta_{s} f$ is almost automorphic (no longer assumed uniformly continuous). Then $f$ is uniformly continuous and almost automorphic.

It is clear that if $f_{\varepsilon} C(\mathrm{R})$ then the set of $t$ such that $\Delta_{t} f$ is uniformly continuous forms a group. Hence our requirement that $T$ be dense is not very restrictive since we can always take $T$ to be a group. Similarly, the set of $t_{0}$ such that $\Delta_{t} f$ is almost automorphic forms a group.

Our main technical device is Lemma 3 which describes a strange sort of scattered equicontinuity of the set of functions $\left\{\Delta_{t} f\right\}$. The mild recurrence relation of almost automorphy on some $\Delta_{s} f$ then forces uniform continuity of $f$. With stronger hypotheses on the group $T$ we may dispense with the recurrence relation. For example, if $T$ is of second category in $R$, then $f$ will

This work was partially supported by N.S.F. Grant NSF GP 5370. 
be uniformly continuous regardless of any recurrence relations. However, it is possible to have a dense subset $T$ and $f \varepsilon C(\mathbf{R})$ so that $\Delta_{t} f$ is uniformly continuous for each $t \varepsilon T$ and yet $f$ is not uniformly continuous, so in general, some recurrence relation is necessary.

Finally, we present an illustrative example of a function $f_{\varepsilon} C(\mathbf{R})$ such that $\Delta_{1} f$ is almost periodic but such that $f$ is not almost periodic; indeed, $f$ is not even almost automorphic.

This example represents a joint effort of the author and K. B. Fine. We take pleasure in acknowledging valuable discussions with Dr. Fine.

$\S 1$. We first recall the definitions of almost periodicity and almost automorphy.

We call $f \varepsilon C(\mathbf{R})$ almost periodic if for each sequence of real numbers $\left\{t_{n}^{\prime}\right\}$ there exists a subsequence $\left\{t_{n}\right\}$ such that $\left\{f\left(x+t_{n}\right)\right\}$ converges uniformly. This definition is equivalent to H. Bohr's definition, by a well known result of Bochner [1].

The definition of almost automorphy is also due to Bochner [1], but is possibly not as familiar. We call $f \varepsilon C(\mathbf{R})$ almost automorphic (a.a.) if for each sequence of real numbers $\left\{t_{n}^{\prime}\right\}$ there exists a subsequence $\left\{t_{n}\right\}$ such that for each $x$ we have $\lim _{m} \lim _{n} f\left(x+t_{n}-t_{m}\right)=f(x)$.

If $f$ is uniformly continuous and almost automorphic (what W. A. Veech calls continuously almost automorphic) we say $f$ is u.c.a.a. We often call such a function a.a. if the uniform continuity is obvious.

It is clear that almost periodicity implies uniform continuity and almost automorphy.

W. A. Veech has obtained fundamental results on almost automorphic functions. We shall not need any of his deeper results in this note. However, we owe to Veech [3], [4] the result that the concepts of almost periodicity, almost automorphy, and continuous almost automorphy, which we treat here as distinct, are indeed distinct.

It is now convenient to introduce some special notation for moduli of continuity.

If $f \varepsilon C(\mathrm{R})$ we define $\eta(f)$ by

$$
\eta(f)=\inf \{\epsilon|\exists \delta:| x-y|<\delta \Rightarrow| f(x)-f(y) \mid<\epsilon\} .
$$

Clearly $\eta(f)=0$ if and only if $f$ is uniformly continuous.

If for all $x$ and $y$ in some set $I$ of reals we have $|x-y| \leqq \delta \Rightarrow|f(x)-f(y)| \leqq \epsilon$, we call $\delta$ an $\epsilon$-modulus of continuity on $I$.

We denote the length of the interval $I$ by $l(I)$.

Finally, we define $\operatorname{osc}_{I} f$, the oscillation of $f$ on $I$, by $\operatorname{osc}_{I} f=\sup _{x, y \varepsilon I}|f(x)-f(y)|$.

Lemma 1. Let $f \varepsilon C(\mathrm{R})$ be almost automorphic. Let $\epsilon>0$ and $\delta>0$ be fixed. Suppose that for each $M>0$ there is an interval $I_{M}$ of length greater than $M$ such that $\delta$ is an $\epsilon$-modulus of continuity on $I_{M}$. Then $\delta$ is an $\epsilon$-modulus of continuity on all of $\mathbf{R}$. 
Proof. Select a sequence $\left\{\alpha_{n}\right\}$ such that $\alpha_{n}$ is the midpoint of $I_{n}$ where $l\left(I_{n}\right)>n$. Then for any subsequence $\alpha_{n}^{\prime}$ such that the pointwise limit exists we see that $g(x) \equiv \lim _{n} f\left(x+\alpha_{n}^{\prime}\right)$ has $\delta$ as an $\epsilon$-modulus everywhere. But then for any sequence $\beta_{n}$ where the pointwise limit exists we see that $h(x) \equiv \lim _{m}$ $g\left(x+\beta_{m}\right)$ similarly has $\delta$ as an $\epsilon$-modulus everywhere. In particular if $\beta_{m}=\alpha_{m}^{\prime \prime}$ is a subsequence of $\alpha_{m}^{\prime}$ we see that $f(x)=\lim _{m} \lim _{n} f\left(x+\alpha_{n}^{\prime \prime}-\alpha_{m}^{\prime \prime}\right)$ has the desired property.

Lemma 2. Let $f \varepsilon R(\mathrm{R})$. Assume $f$ is not uniformly continuous. Let $T$ be a dense set such that if $t \varepsilon T$ then $\Delta_{t} f$ is uniformly continuous. Then if $\eta_{1}<\eta(f)$, $\delta>0$, and $M>0$ are given there is an interval $I_{M}$ such that $l\left(I_{M}\right)>M$ and such that if $J$ is a subinterval of $I_{M}$ and $l(J)>\delta$ then $\operatorname{osc}_{J} f>\eta_{1}$.

Proof. Suppose, for the sake of contradiction, that there exists an $\eta_{1}<\eta(f)$, an $M>0$ and $\delta>0$ with the property that each interval of length greater than $M$ has a subinterval $K$ of length greater than $\delta$ such that $\operatorname{osc}_{K} f \leqq \eta_{1}$. Call the set of such subintervals $\left\{K_{i}\right\}$. Because $f$ is not uniformly continuous there exists an $\eta_{2}>\eta_{1}$ with the property that for any $n$ there is a subinterval $J_{n}$ such that $l\left(J_{n}\right)<\delta / n$ and $\operatorname{osc}_{J_{n}} f>\eta_{2}$. Hence there exists some interval $I$ such that for each $t \varepsilon I$ and for some infinite set of $n$ we have $J_{n}+t \subset K_{i}$ for some $i$. Hence $\operatorname{osc}_{J_{n+t}}(f(x+t)-f(x)) \geqq \eta_{2}-\eta_{1}$.

Since $l\left(J_{n}\right) \rightarrow_{n} 0$ we see that if $t \varepsilon I$ then $\Delta_{t} f$ is not uniformly continuous. This contradicts the denseness of $T$, and completes our proof.

Our next lemma, which is crucial, establishes a sort of scattered equicontinuity for the functions described in that it establishes for large intervals a modulus of continuity for $\Delta_{t} f$, where this modulus is independent of $t$ but where the location of the interval depends on $t$. The conclusion of the lemma holds trivially if $f$ is uniformly continuous, but that case is of no interest.

Lemma 3. Let $f \varepsilon R(\mathbf{R})$. Let $f$ not be uniformly continuous. Let $T$ be a dense set such that if $t \varepsilon T$ then $\Delta_{t} f$ is uniformly continuous. Let $\epsilon>0$ be given. Then there is $a \delta>0$ such that for each $t \varepsilon T$ and $M>0$ there is an interval $I_{M, t}$ of length greater than $M$ with the property that $\delta$ is an $\epsilon$-modulus on $I_{M, t}$ for the function $\Delta_{t} f$.

Proof. Let $\epsilon, M, t \varepsilon T$ be given. Choose $V=[-2 \delta, 2 \delta]$ so that if $|x-y| \leqq 4 \delta$ then $|f(x)-f(y)|<\eta(f)+\epsilon$. Choose $V_{1}=\left[-\delta_{1}, \delta_{1}\right]$ so that $\delta_{1}<\delta$ and so that if $|x-y|<2 \delta_{1}$ then $\left|\Delta_{t} f(x)-\Delta_{t} f(y)\right|<\epsilon$. By Lemma 2 we can choose an interval $I$ such that $l(I)>M$ and an interval $I_{1}$ generously containing $I$ (say $\left.I_{1}=I+\left[-\left(|t|+\delta+\delta_{1}\right),\left(|t|+\delta+\delta_{1}\right)\right]\right)$ such that if $J$ is a subinterval of $I_{1}$ and $l(J)>\delta_{1} / 2$ then $\operatorname{osc}_{J} f>\eta-\epsilon$.

We now fix $x \varepsilon I$.

We observe that $\operatorname{osc}_{x+v} f<\eta+\epsilon$.

Now if we choose $y$ so that $y+V_{1} \subset x+V$ then $\operatorname{osc}_{y+V_{1}} f>\eta-\epsilon$.

Hence 


$$
\begin{aligned}
\eta-\epsilon< & \sup _{x+V} f-\inf _{x+V} f<\eta+\epsilon, \\
& \sup _{x+V} f-\sup _{y+V_{1}} f<2 \epsilon, \\
& \inf _{x+V} f-\inf _{y+V_{2}} f>-2 \epsilon .
\end{aligned}
$$

The same inequalities hold when we replace $x$ and $y$ by $x+t$ and $y+t$ respectively.

Now we choose $y^{\prime}$ in $y+V_{1}$ so that $f$ attains its maximum in $y+V_{1}$ at $y^{\prime}$ and choose $y^{\prime \prime}$ in $y+V_{1}$ for a similar minimum point. Then

$$
\begin{aligned}
& \Delta_{t} f\left(y^{\prime}\right) \leqq \sup _{y+t+V_{1}} f-f\left(y^{\prime}\right) \leqq \sup _{x+V+t}-\sup _{x+V} f+4 \epsilon \\
& \Delta_{t} f\left(y^{\prime \prime}\right) \geqq \inf _{x+V+t} f-\inf _{x+V} f-4 \epsilon \geqq \sup _{x+V+t} f-\sup _{x+V} f-8 \epsilon .
\end{aligned}
$$

But since $\left|\Delta_{t} f\left(y^{\prime \prime}\right)-\Delta_{t} f\left(y^{\prime}\right)\right|<\epsilon$ we see that

$$
\left|\Delta_{t} f\left(y^{\prime \prime}\right)-\left(\sup _{x+V+t} f-\sup _{x+V} f\right)\right|<9 \epsilon
$$

and so we see that

$$
\left|\Delta_{t} f(y)-\left(\sup _{x+V+t} f-\sup _{x+V} f\right)\right|<10 \epsilon .
$$

Since $y$ could be any point in $[-\delta, \delta]+x$ our proof is complete.

Theorem 4. Let $f \varepsilon C(\mathrm{R})$ and let $T$ be a dense set such that if $t \varepsilon T$ then $\Delta_{t} f$ is uniformly continuous. Let there be a non-zero $t_{0} \varepsilon T$ such that $\Delta_{t_{0}} f$ is almost automorphic. Then $f$ is uniformly continuous.

Proof. We can assume without loss of generality that $f \varepsilon R(\mathrm{R})$ because both the real and the imaginary parts of an a.a. function are a.a. We can assume that $t_{0}=1$. We also assume, for the sake of contradiction, that $f$ is not uniformly continuous. Now we choose $\epsilon<\eta(f)$. We then note that for any integer $n$ the function $\Delta_{n} f$ is u.c.a.a. However, by Lemma 2 we can choose for any $\epsilon^{\prime}>0$ and any $\delta>0$ an $n$ such that $\operatorname{osc}_{n+\{-\delta / 2, \delta / 2\}} f>\eta-\epsilon^{\prime}$. Hence it is clear that for any $\delta$ we can choose an $n$ such that $\operatorname{osc}_{[-\delta / 2, \delta / 2]} \Delta_{n} f>\epsilon$.

But by Lemma 3 there is a $\delta$ such that on arbitrarily long intervals $I_{M}$ we have

$$
|x-y|<\delta \Rightarrow\left|\Delta_{n} f(x)-\Delta_{n} f(y)\right|<\epsilon / 2 .
$$

Hence by Lemma 1,

$$
|x-y|<\delta \Rightarrow\left|\Delta_{n} f(x)-\Delta_{n} f(y)\right| \leqq \epsilon / 2
$$

for all $x, y \varepsilon \mathrm{R}$, which contradicts

$$
\underset{1-\delta / 2, \delta / 2]}{\operatorname{Osc}} \Delta_{n} f>\epsilon
$$

This completes our proof. 
We now give the special case of Bochner's theorem (Theorem 4 of [1]) which we shall use.

Theorem (BochNer). If $f \in C(\mathbf{R})$ is uniformly continuous and there is a non-zero $t$ such that $\Delta_{t} f$ is a.p. (a.a.) then $f$ is a.p. (a.a.).

Theorem 4 and Bochner's theorem immediately yield the following corollary.

Corollary 5. Let $f \varepsilon C(\mathbf{R})$. Suppose that there is a dense set $T$ such that if $t \varepsilon T$ then $\Delta_{t} f$ is uniformly continuous. Suppose that there exists a non-zero $t_{0} \varepsilon T$ such that $\Delta_{t_{0}} f$ is a.p. (a.a.). Then $f$ is a.p. (u.c.a.a.).

This result settles in the affirmative one of the author's original questions: that is, if $f \varepsilon C(\mathbf{R})$ and $\Delta_{t} f$ is a.p. for each rational $t$ then is $f$ itself a.p.? However this particular question is settled more easily by Loomis's Theorem 3 of [2] which can be easily modified to work for a dense set of translates. Our thanks are due to R. Kaufman for directing our attention to this theorem.

The modified form follows.

Theorem (Loomis). Let $f \in C(\mathbb{R})$. Suppose that there is a dense set $T$ such that if $t \varepsilon T$ then $\Delta_{t} f$ is almost periodic. Then $f$ is almost periodic.

We could then use Loomis's theorem with Bochner's theorem to get Corollary 5 in the case of almost periodicity. Indeed, if $\Delta_{t} f$ is uniformly continuous and $\Delta_{t_{0}} f$ is a.p. then, since $\Delta_{t_{0}}\left(\Delta_{t} f\right)=\Delta_{t}\left(\Delta_{t_{0}} f\right)$, Bochner's theorem yields the almost periodicity of $\Delta_{t} f$. Hence the hypotheses of Corollary 5 imply the hypotheses of Loomis's theorem.

The following extension is now easy to prove.

Corollary 6. Let $f \varepsilon C(\mathbf{R})$. Let $T$ be a dense subset of $R$ such that if $t \varepsilon T$ then $\Delta_{t} f$ is uniformly continuous. Suppose there is a real non-zero s such that $\Delta_{s} f$ is almost automorphic (no longer assumed uniformly continuous). Then $f$ is uniformly continuous and almost automorphic.

Proof. Since $\Delta_{s}\left(\Delta_{t} f\right)=\Delta_{t}\left(\Delta_{s} f\right)$ we see that if $t \varepsilon T$ then $\Delta_{s}\left(\Delta_{t} f\right)$ is uniformly continuous and almost automorphic. Hence the uniformly continuous function $\Delta_{t} f$ is itself almost automorphic and so, by Corollary 5 , we see that $f$ is uniformly continuous and almost automorphic.

Proposition 7. Let $f \varepsilon C(\mathbf{R})$. Suppose that there is a set $T$ of the second category in $\mathbf{R}$ such that $\Delta_{t} f$ is uniformly continuous for each $t \varepsilon T$. Then $f$ is uniformly continuous.

Proof. Assume $f$ is not uniformly continuous. Choose $\epsilon<\eta(f) / 2$. If $\Delta_{t_{0}} f$ is uniformly continuous, then for any neighborhood of $t_{0}$ and any $\delta>0$ there exists a $t_{1}$ in that neighborhood so that $\delta$ will not be an $\epsilon$-modulus for $\Delta_{t_{1}} f$. Indeed, by Lemma 2, we have an interval on which $f$ has rapid oscillations of period less than $\delta$ and amplitude greater than $2 \epsilon$. Also, for this interval $f$ is approximately duplicated to within an additive constant on the interval 
translated by $t_{0}$. Hence we may choose a small perturbation to some $t_{1}$ so as to bring peaks over valleys and cause $\delta$ to fail as an $\epsilon$-modulus for $\Delta_{t_{1}} f$. The details are similar to those of Lemma 3. It is clear now that for real $s$ close enough to $t_{1}$ we cannot have $\delta$ as an $\epsilon$-modulus for $\Delta_{s} f$. Hence for each $\delta$ the set of $t$ such that $\delta$ serves as an $\epsilon$-modulus for $\Delta_{t} f$ is nowhere dense and so, letting $\delta$ run over the positive rationals, we see that $T$ is first category in $R$. This completes our proof.

It is easy to construct an example of a dense $T$ and $f \varepsilon C(\mathbf{R})$ such that $\Delta_{t} f$ is uniformly continuous for each $t \varepsilon T$ and yet $f$ is not uniformly continuous.

In the case, for example, where $T$ is the set of rationals we can let $f$ be the pointwise sum of an infinite set of $f_{n}$, each $f_{n}$ of finite support, the supports disjoint, and the modulus of continuity for $\epsilon=1$ going to infinity in $n$. In addition we can make $f_{n}$ oscillate sufficiently rapidly and regularly while yet reducing the oscillations towards the ends of its support interval so that $\left\|\Delta_{r / n} f_{n}\right\|_{\infty}<1 / 2^{n}$ for $r=1, \cdots, n$ !. It is clear that such an $f$ will serve. Indeed, for each rational $t$ we see that $\lim _{x \rightarrow \infty} \Delta_{t} f(x)=0$.

It is not hard to make up more complicated examples so that the scattered equicontinuity guaranteed by Lemma 3 is essentially all we have.

We do not know, however, whether there is such a construction for each first category group.

We now construct our $f \varepsilon C(\mathbf{R})$ such that $f$ is not almost automorphic but $\Delta_{1} f$ is almost periodic. We shall define $f$ as the pointwise limit of a series of periodic functions $f_{n}$.

The function $f_{n}$ will be a real valued continuous function of period $2^{n+1}$. The graph of $f_{n}$ consists of a sequence of isoceles triangles based on the intervals $\left[m-2^{-n+1}, m-2^{-n}\right]$. The altitudes of these triangles taper linearly from 0 on $\left[1-2^{-n+1}, 1-2^{-n}\right]$ to 1 on $\left[\left(2^{n}+1\right)-2^{-n+1},\left(2^{n}+1\right)-2^{-n}\right]$ and then taper back down to $2^{-n}$ on the interval $\left[2^{n+1}-2^{-n+1}, 2^{n+1}-2^{-n}\right]$. The periodicity requirement completes the definition of $f_{n}$. (Between triangles we take $f_{n}$ to be 0 .) We note that if $m \neq n$, then for any $x$ if $f_{m}(x) \neq 0$ then $f_{n}(x)=0$.

Now we claim that $f(x)=\sum_{n=1}^{\infty} f_{n}(x)$ is the desired function. We see that $\Delta_{1} f$ is a.p. Indeed, we can write $\Delta_{1} f$ as the uniform limit of the periodic functions $\sum_{n=1}^{r} \Delta_{1} f_{n}$ where we observe that $\left\|\Delta_{1} f_{n}\right\|_{\infty}=2^{-n}$. It is clear that $f \varepsilon C(\mathbf{R})$, yet $f$ is not a.p. Indeed, $f$ is not uniformly continuous.

It is only slightly more difficult to see that $f$ is not a.a. Let $\alpha_{n}=2^{n}+3 / 2^{n+1}$. Then $\lim _{m} \lim _{n} f\left(1+\alpha_{n}-\alpha_{m}\right)=1$. To see that the above limit is 1 we observe that $f\left(1-\alpha_{m}\right)$ is $f$ evaluated at the peak of the highest triangle of the periodic function $f_{m}$. But for large enough $n$, it is clear that $1+\alpha_{n}-\alpha_{m}$ represents a translate of the argument $1-\alpha_{m}$ by a large multiple of $2^{m+1}$, the period of $f_{m}$, plus a small perturbation. Since for such $m, n$ we see that $f\left(1+\alpha_{n}-\alpha_{m}\right)=$ $f_{m}\left(1+\alpha_{n}-\alpha_{m}\right)$ it is clear that $\lim _{n} f\left(1+\alpha_{n}-\alpha_{m}\right)=1$ for each $m$. Now, since $f(1)=0$, we see that $\lim _{m} \lim _{n} f\left(1+\alpha_{n}-\alpha_{m}\right) \neq f(1)$, and since this inequality is preserved under subsequences we see that $f$ is not a.a. 


\section{REFERENCES}

[1] S. A. Bochner, A new approach to almost periodicity, Proc. N.A.S., 48 (1962) 2039-2043.

[2] L. H. Loomis, The spectral characterization of a class of almost periodic functions, Ann. of Math., 72 (1960) 362-368.

[3] W. A. VeECH, Almost automorphic functions on groups, Amer. J. Math., 87 (1965) 719-751.

[4] —, On a theorem of Bochner, Ann. of Math., 86 (1967) 117-137.

University of Illinois

Urbana, Illinois

Date communicated: FEBRUARY 12, 1969 